\title{
Controlled Manipulation of Atoms in Insulating Surfaces with the Virtual Atomic Force Microscope
}

\author{
T. Trevethan, ${ }^{1}$ M. Watkins, ${ }^{1}$ L. N. Kantorovich, ${ }^{2}$ and A. L. Shluger ${ }^{1}$ \\ ${ }^{1}$ Department of Physics and Astronomy, University College London, Gower Street, London WC1E 6BT, United Kingdom \\ ${ }^{2}$ Department of Physics, King's College London, Strand, London WC2R 2LS, United Kingdom
}

(Received 24 February 2006; published 8 January 2007)

\begin{abstract}
We predict how single oxygen ions can be manipulated on the $\mathrm{MgO}$ (100) surface and demonstrate the possibility of detecting a single-atom event using a noncontact atomic force microscope. The manipulation process is simulated explicitly in real time with a virtual dynamic atomic force microscope including the full response of the instrumentation and demonstrates a strong dependence on temperature. The proposed new atomistic mechanism and protocols for the controlled manipulation of single atoms and vacancies on insulating surfaces may be relevant for anchoring molecules and metal clusters at these surfaces and controlling their electronic properties.
\end{abstract}

PACS numbers: 87.64.Dz, 07.79.Lh, 68.37.Ps

Initiating, controlling, and detecting single atomic scale events using macroscopic instruments, such as scanning probes, is vital for demonstrating the feasibility of molecular electronics and quantum computing as well as engineering and studying elementary surface processes. The scanning tunneling microscope (STM) has wellestablished success in this area [1] but is limited to conducting systems. Contact mode atomic force microscopy (AFM) [2] has been used for pushing and pulling larger nanoscale objects on surfaces (see, for example, $[3,4]$ ); however, controlled reproducible manipulation of surface atoms with the $0.1 \mathrm{~nm}$ precision attained by STM on metals [1] can only be achieved using noncontact AFM (NCAFM) [2]. Very recently lateral atomic manipulation has been achieved using this technique on semiconductor surfaces [5-7]. Atomistic mechanisms of AFM manipulation of adsorbed atoms [8] and defects [9] have been simulated; however, these static calculations only determined potential energy surface (PES) for the manipulation of a species during a single approach of the tip. In this Letter we go further and consider the process of manipulation dynamically and at several temperatures. A direct link with the experimental manipulation procedure is provided using a recently developed virtual atomic force microscope (VAFM) [10,11], explicitly simulating the operation and dynamical response of the entire NC-AFM experimental setup. The results of these calculations for the first time predict experimental signals that can be expected from a single-atom manipulation event and suggest an atomistic mechanism and protocol for the controlled manipulation of atoms at insulating surfaces.

To attain true atomic resolution in noncontact mode AFM, the cantilever oscillates close to its resonant frequency $(10-300 \mathrm{kHz})$ with a constant amplitude (10$30 \mathrm{~nm}$ ). The energy stored in the cantilever oscillations prevents a mechanical jump into contact due to the strong interaction between the tip and surface at small distances. This means the tip interacts strongly with the surface only periodically and for a small fraction of the tip oscillation cycle $\left(\approx 10^{-7} \mathrm{~s}\right)$ when it is close to the surface. To be successful, the desired manipulation event must have a significant probability of occurring during this short time. We demonstrate that it is possible for the tip to reduce or completely remove the energy barrier for the manipulation when it is close to the surface. The system can then move into a new position within the short period of tip proximity. This entirely changes the force field for the interaction of the tip with the surface and should manifest itself in measurable experimental signatures. Similar mechanisms and signals should also apply to adsorbed atoms and small molecules.

To introduce the new mechanism and protocol of manipulation we consider a realistic and potentially important system - a doubly charged oxygen vacancy on the $\mathrm{MgO}$ (100) surface. Vacancies on insulating surfaces can be used for anchoring molecules [12], and on thin films the charge of anion vacancies can be manipulated by applying an appropriate bias between tip and sample [13]. Together with lateral manipulation this makes them a particularly versatile tool for controlling the electronic and chemical properties of adsorbed molecules [14]. Successful manipulation experiments have so far been carried out by laterally scanning the surface area to achieve the desired movement. The manipulation protocol discussed here involves initial imaging of the surface to establish the direction and plan of manipulation and then a series of vertical displacements into particular positions of manipulation until the destination is reached (see Fig. 1), accompanied by a final image. To successfully accomplish manipulation using this protocol one needs to have information about the most favorable conditions for manipulation and clearly identifiable signals that the manipulation has been accomplished at each stage.

To simulate the process of manipulation we consider the $\mathrm{MgO}$ (100) surface interacting with a $\mathrm{MgO}$ tip, which has been shown to be a good model for a wide class of polar tips [15]. To calculate the tip-surface interaction, the gen- 


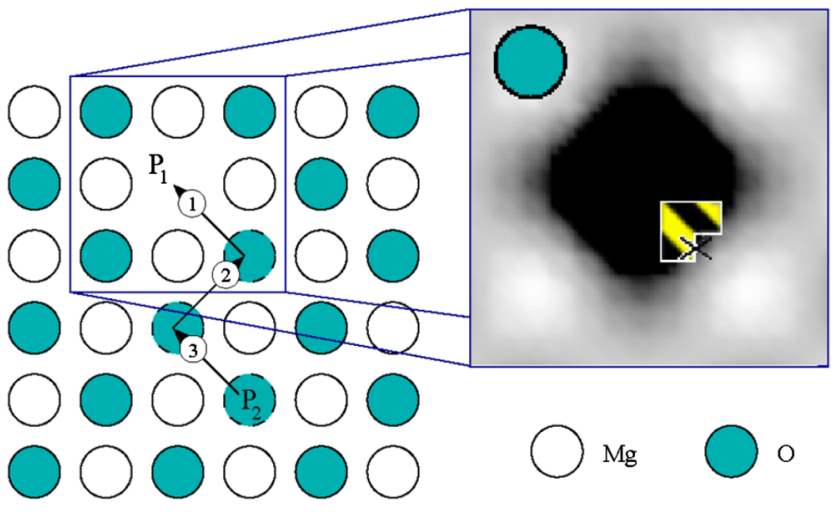

FIG. 1 (color online). Illustration of the procedure for manipulating an oxygen vacancy to any desired location on the surface. In this example the vacancy is moved from position $P_{1}$ to position $P_{2}$ by approaching with the oscillating tip at the lateral positions indicated, in the sequence 1-2-3. A topography image of the vacancy is also shown as an inset, with the required lateral region for successful manipulation indicated [18]. The point addressed in detail in this Letter is marked with an X.

eral utility lattice program (GULP) atomistic simulation code [16] was employed using shell model pair potentials [17] for the $\mathrm{MgO}$ surface and tip. The $\mathrm{MgO}$ (100) surface is modeled by a periodically repeated array of $16 \times 16 \times 6$ atoms with the lowermost 3 atomic layers frozen in bulklike positions. The tip is represented by a $\mathrm{Mg}$ terminated 64 atom $\mathrm{MgO}$ cube oriented with its threefold axis perpendicular to the surface plane [17] with the uppermost 32 atoms frozen.

A NC-AFM image of the double charged oxygen vacancy on the $\mathrm{MgO}$ surface simulated using the Mg terminated $\mathrm{MgO}$ tip is shown in Fig. 1. We have recently shown how such a vacancy can be laterally manipulated on the $\mathrm{MgO}$ (100) surface using a polar tip [18]. At close approach the localized electric-field gradient of the tip can be used to influence specific surface sites. It was found that an oxygen atom can be moved into a neighboring vacancy by approaching close above the vacancy with the Mg terminated $\mathrm{MgO}$ tip, as illustrated in Figs. 1 and 2. When the tip is far from the surface, the barrier is approximately $1.3 \mathrm{eV}$. As the tip approaches, this barrier is reduced until, at a certain tip height, it disappears [see Fig. 2(b)]. The closest oxygen then moves into the vacancy and remains there as the tip retracts and the barrier increases again.

The PES [see Fig. 2(a)] of the vacancy manipulation for a given fixed tip position above the surface is determined using a constrained minimization scheme fully described in [18]. To facilitate the jump of one out of four equivalent oxygen ions, the lateral position of the tip should be shifted from the position directly above the vacancy towards the oxygen ion that it is intended to move (see Fig. 1). For the tip locations that lead to successful manipulation the lateral distance to the target oxygen from the tip apex was at least $0.9 \AA$ smaller than the distance to the other three oxygen ions that could be manipulated. Calculations of the energy
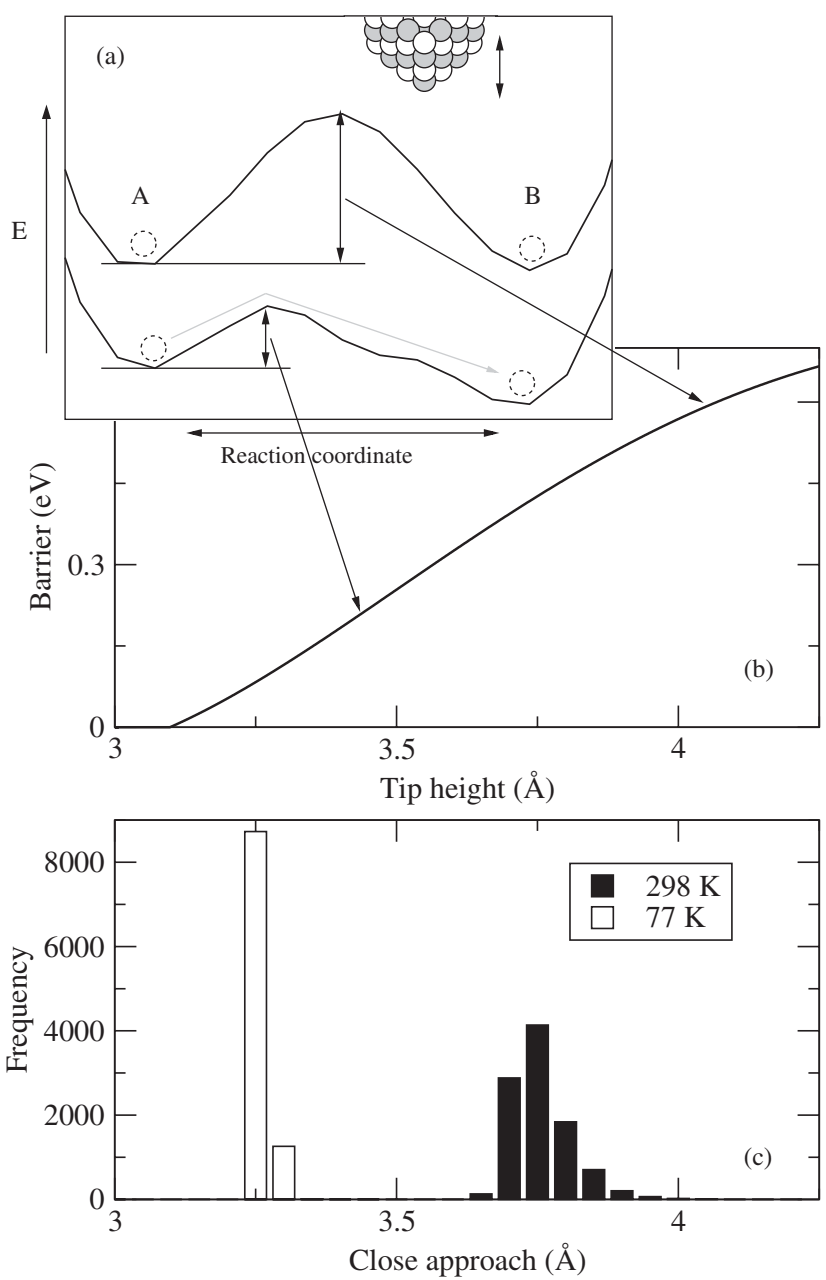

FIG. 2. (a) Potential energy curves for two tip heights (3.4 and $4.5 \AA$ ). (b) Energy barrier to cross from state $A$ into states $B$ or $C$. (c) Example distributions of the tip height at which the jump occurs for 77 and $298 \mathrm{~K}$, for 10000 attempts.

barriers for various tip positions and heights show that it is virtually certain that, if the tip is located within the area highlighted in Fig. 1, the desired manipulation event will occur, rather than tip induced diffusion of any other surface $\mathrm{O}$ ion. These locations cover an area of approximately $0.75 \AA^{2}$ and would allow a conservative error of $\pm 0.25 \AA$ to occur in the lateral positioning of the tip while still being almost certain of inducing the desired manipulation event.

For a fixed lateral tip position, shifted by a distance $1.12 \AA$ in the $(100)$ direction and $0.88 \AA$ in the $(010)$ towards the target oxygen from the lateral position directly above the vacancy (see Fig. 1), the calculation for the potential energy surface of the vacancy manipulation is repeated for a series of vertical tip heights in the range 2.5$8 \AA$ in steps of $0.1 \AA$ [the potential energy curves for tip heights 3.4 and $4.5 \AA$ are shown in Fig. 2(a)]. From this series of curves the energies of the minima and the barriers that separate them are then determined as a function of tip height. In further discussion state $A$ corresponds to the 
initial position of the vacancy before the $\mathrm{O}$ ion has jumped, and state $B$ arises when the $\mathrm{O}$ ion has moved into the vacancy. An additional state $C$ [not shown in Fig. 2(a)] arises due to the jump of the $\mathrm{O}$ ion onto the tip apex when the tip is very close to the surface (see, for example, [19]). The two separate states, $B$ and $C$, and the barrier separating them are a consequence of the overlap of the potential wells for the $\mathrm{O}$ ion adsorption on the tip apex and location in the surface site [19]. We have shown previously that the system moving from state $B$ to state $C$ and then back to $B$ will result in a structurally reversible hysteresis in the tip force and hence an energy dissipation channel for each subsequent oscillation cycle at close approach [20].

The point at which the system will jump from state $A$ over the barrier and its evolution between states $B$ and $C$ will depend on the tip height, the cantilever dynamics, and the temperature of the system. Positioning the tip in the successful manipulation area shown in Fig. 1, the barrier for the system to return to its initial state $A$ is much higher [see Figs. 2(a) and 2(b)]. This makes state $A$ inaccessible after the manipulation event, confining the system to states $B$ or $C$ depending on tip height, resulting in irreversible and controlled manipulation.

Manipulation consists of a single $\mathrm{O}$ ion jump, which is then detected by the NC-AFM electronics. Unlike in previous works [8,9], we treat this event statistically and explicitly take into account that the probability of each jump depends on temperature, the barrier height (and therefore the tip height), and the tip velocity. For a given temperature, cantilever approach velocity and cantilever oscillation frequency, the distribution and expectation value of the point in time at which this jump occurs is determined via a kinetic Monte Carlo method [21]. In this calculation the transition rate at a certain tip height is determined as in [20], and used to obtain the probability of the jump occurring within a short time step. A random number is then produced to evaluate whether the transition occurs at this point. The approach trajectory is repeated many times to build up a distribution of the distance of closest approach and cycle number at which the jump occurs. Distributions of tip heights at which the jumps occur for two temperatures are shown in Fig. 2(c). It is interesting to note that the higher the temperature the earlier the jump occurs and the broader the distribution of tip height. The expectation value of this distribution is then used as the point of the jump in the VAFM simulations. Once this single jump has occurred, the evolution of the system between states $B$ and $C$ and the resulting tip-surface interaction is determined using the dynamical response method described in [20].

The manipulation jump results in a permanent structural change leading to a sudden change in the force field experienced by the tip over its trajectory. There is no hysteresis in the tip-surface force over oscillations before the jump, a large hysteresis in the tip-surface force in the oscillation in which the jump takes place $(1-3 \mathrm{eV})$, and no (or small, $\leq 0.1 \mathrm{eV}$ ) hysteresis after the jump has oc- curred. The hysteresis in the tip-surface force during the manipulation event means that energy is dissipated from the driven cantilever into the surface. To maintain the amplitude of the cantilever oscillations, energy must be fed back into the cantilever. The magnitude of this excitation is monitored and gives the damping signal.

To investigate the effect that the manipulation event has on the instrument and predict the signals that will be observed experimentally, the VAFM is employed to simulate a vertical approach over the lateral point described above. In the VAFM simulation the cantilever $\left(f_{0}=\right.$ $160 \mathrm{kHz}$ and $k=20 \mathrm{~N} \mathrm{~m}^{-1}$ ) is initially set oscillating at a distance of closest approach $5 \AA$ from the surface and at the amplitude set point of $A_{0}=10 \mathrm{~nm}$. In addition to the microscopic tip-surface interaction, a macroscopic van der Waals force, with a tip radius of $5 \mathrm{~nm}$, is added to obtain the total force on the tip. This gives a normalized frequency shift $\gamma=8.75 \mathrm{fN} \mathrm{m}^{1 / 2}$, which is representative of typical experimental setups [5-7].

Figure 3 shows the frequency shift, closest approach, and the cantilever excitation signal as a function of cantilever oscillation cycle number (from the point at which the approach started at $5 \AA$ ) for the distance control approaching the frequency shift set point of $\Delta f_{0}=-70 \mathrm{~Hz}$ at $T=4,77$, and $298 \mathrm{~K}$. Initially the system is in state $A$ as the surface is approached. Then the system jumps from state $A$ to states $B$ or $C$ at a distance of closest approach of approximately $3.1 \AA$ at $4 \mathrm{~K}, 3.3 \AA$ at $77 \mathrm{~K}$, and $3.8 \AA$ at $298 \mathrm{~K}$. At the point of the jump the force field experienced by the tip changes suddenly, which then causes a spike in

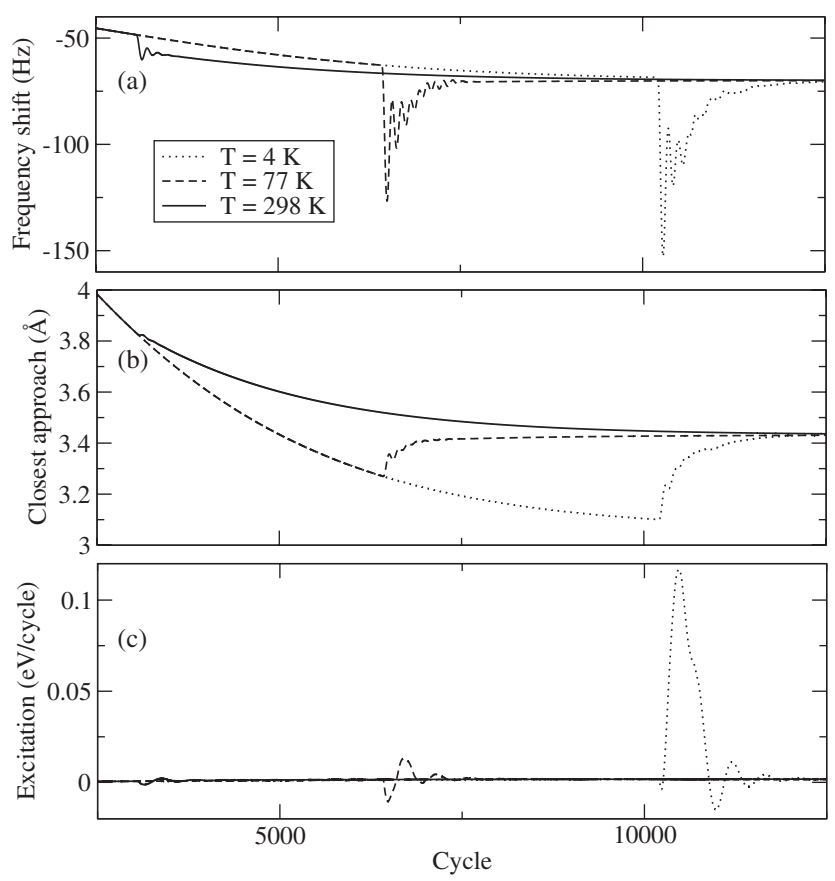

FIG. 3. Experimental signals [(a) frequency shift, (b) closest approach, and (c) dissipation] as a function of cycle when looking for the frequency set point $(-70 \mathrm{~Hz})$ below the manipulation point for the temperatures of 4,77 , and $298 \mathrm{~K}$. 
the frequency shift over the following cycles. In the case of the $T=4$ and $77 \mathrm{~K}$ simulations, this rapid decrease in frequency causes the distance control to retract the cantilever from the surface, to maintain the frequency set point. When the jump occurs, it is followed by a spike in the excitation signal which is due to energy being lost by the cantilever due to the hysteresis in the tip-surface force over the single oscillation cycle of the manipulation. The amplitude control increases the excitation signal to return the amplitude of the cantilever oscillations to the set-point value after the loss of this mechanical energy. It then decays to the background level or to a steady state dissipation level depending on the distance of closest approach and the temperature. It is clear from the signals in Fig. 3 that the process strongly depends on the temperature. As the temperature is increased, the system can cross the barrier at a greater tip-surface separation (see Fig. 2), resulting in both a smaller hysteresis and smaller change in the tip-surface force field.

The types of experimental signatures shown in Fig. 3 (the jump in the frequency shift and the spike in the excitation signal) indicate that the manipulation has successfully been achieved. Once these signals have been seen the cantilever can be retracted and the next stage of the experiment, i.e., the next manipulation, can be carried out without having to rescan the surface. In this case, given that the lateral position can be specified precisely enough and that thermal drift can be sufficiently negated [22]), this procedure can be repeated to move the vacancy anywhere on the surface relatively quickly and without the need to image the surface at each step to check that the vacancy has been successfully moved. The results of the VAFM calculations in Fig. 3 show that the signatures will be more pronounced at lower temperatures; however, the tip must get much closer to the surface in order to cause the manipulation.

In order to have sufficient control over the manipulation process it is important to have accurate knowledge of the distance of closest approach that is required to cause the vacancy jump. This will be approximately the distance at which atomic scale dissipation will be observed on the ideal surface. An alternative method to calibrate the distance of closest approach involves measuring the forcedistance curves above different atoms on the surface. These can then be used to eliminate the long range component of the tip-surface force and the separation can be determined by comparison with atomistic simulations [23].

To summarize, we have simulated the process of atomic manipulation using a realistic model for the NC-AFM instrument and demonstrated how the position of a vacancy on an oxide surface can be moved in a controlled manner. The experimental signals that were obtained correspond to registering a single-atom event with the NC-AFM. The method described in this Letter will be directly applicable to manipulation of vacancies on alkali halide and other cubic oxide surfaces. The same approach to modeling AFM manipulation should be applicable to many other systems where a structural change is induced by interaction with the tip in NC-AFM and where this structural change results in a significantly different force field for the tip for a given lateral position.

This work was supported through the EU FP6 projects "Nanoman" and "PICO-inside." The authors are grateful to O. Custance, R. Perez, J. Polesel-Maris, S. Gauthier, M. Sterrer, and M. Reichling for valuable discussions.

[1] D. Eigler and E. Schweizer, Nature (London) 344, 524 (1990).

[2] F. J. Giessibl, Rev. Mod. Phys. 75, 949 (2003).

[3] T. Junno, S. Carlsson, H. Xu, L. Montelius, and L. Samuelson, Appl. Phys. Lett. 72, 548 (1998).

[4] H. Postma, A. Sellmeijer, and C. Dekker, Adv. Mater. 12, 1299 (2000).

[5] N. Oyabu, O. Custance, I. Yi, Y. Sugawara, and S. Morita, Phys. Rev. Lett. 90, 176102 (2003).

[6] N. Oyabu, Y. Sugimoto, M. Abe, O. Custance, and S. Morita, Nanotechnology 16, S112 (2005).

[7] Y. Sugimoto, M. Abe, S. Hirayama, N. Oyabu, O. Custance, and S. Morita, Nat. Mater. 4, S156 (2005).

[8] L. Pizzagalli and A. Baratoff, Phys. Rev. B 68, 115427 (2003).

[9] P. Dieska, I. Stich, and R. Perez, Phys. Rev. Lett. 95, 126103 (2005).

[10] J. Polesel-Maris and S. Gauthier, J. Appl. Phys. 97, 044902 (2005).

[11] L. N. Kantorovich, J. Polesel-Maris, and T. Trevethan, virtual atomic force microscope simulation code. See EPAPS Document No. E-PRLTAO-98-020702 for the manual and further information. For more information on EPAPS, see http://www.aip.org/pubservs/epaps.html.

[12] M. Stengel, A. De Vita, and A. Baldereschi, Phys. Rev. Lett. 91, 166101 (2003).

[13] M. Sterrer, M. Heyde, M. Novicki, N. Nilius, T. Risse, H. P. Rust, G. Pacchioni, and H. J. Freund, J. Phys. Chem. B 110, 46 (2006).

[14] P. G. Piva, G. A. DiLabio, J. L. Pitters, J. Zikovsky, M. Rezeq, S. Dogel, W. A. Hofer, and R. A. Wolkow, Nature (London) 435, 658 (2005).

[15] W. A. Hofer, A. S. Foster, and A. L. Shluger, Rev. Mod. Phys. 75, 1287 (2003).

[16] J. Gale and A. Rohl, Mol. Simul. 29, 291 (2003).

[17] A. L. Shluger, A. L. Rohl, D. H. Gay, and R. T. Williams, J. Phys. Condens. Matter 6, 1825 (1994).

[18] M. Watkins and A. Shluger, Phys. Rev. B 73, 245435 (2006).

[19] A. L. Shluger, L. N. Kantorovich, A. I. Livshits, and M. J. Gillan, Phys. Rev. B 56, 15332 (1997).

[20] L. Kantorovich and T. Trevethan, Phys. Rev. Lett. 93, 236102 (2004).

[21] T. Trevethan, M. Watkins, L. Kantorovich, A. L. Shluger, J. Polesel-Maris, and S. Gauthier, Nanotechnology 17, 5866 (2006).

[22] M. Abe, Y. Sugimoto, O. Custance, and S. Morita, Appl. Phys. Lett. 87, 173503 (2005).

[23] R. Hoffmann, L. N. Kantorovich, A. Baratoff, H. J. Hug, and H. J. Guntherodt, Phys. Rev. Lett. 92, 146103 (2004). 